\title{
Comparative Study of Pure and Magnesium Doped Cadmium Mercury Thiocyanate Single Crystals - Gel Technique
}

\author{
P. Nisha Santha Kumari ${ }^{\mathrm{a}}$ and S. Kalainathan ${ }^{\mathrm{b}}{ }$ \\ ${ }^{a}$ Department of Physics, Auxilium College, Vellore-632006, India \\ ${ }^{b}$ School of Science and Humanities, VIT University, Vellore-632014, India. \\ *Corresponding Author: E-mail: Kalainathan@yahoo.com
}

\begin{abstract}
Growth of magnesium doped cadmium mercury thiocyanate single crystals from silica gel by the process of diffusion and a comparative study with pure cadmium mercury thiocyanate are discussed. The X-ray diffraction studies reveal the crystal lattice of the doped crystal to be tetragonal showing that the incorporation of the metallic dopant has not altered the structure of the parent crystal. Investigation on the crystalline perfection of the grown crystal by high resolution X-ray diffraction analysis reveals the quality of the doped crystal to be extremely good. Fourier transform infrared and transmission spectra were also recorded for the grown crystals. The thermal stabilities of the crystals were investigated by thermogravimetric and differential thermal analyses. Vickers microhardness studies reveal that the presence of dopant has changed the nature of the crystal from soft to hard material category. The second harmonic generation efficiencies of the crystals were measured with respect to urea.
\end{abstract}

Key words: Crystal growth; Diffusion; X-ray diffraction; Mechanical properties.

\section{INTRODUCTION}

Nonlinear optical materials are of much importance for optical applications. Recently bimetallic thiocyanate complexes of type $\mathrm{AB}(\mathrm{SCN})_{4}$ are found to exhibit excellent nonlinear optical properties which attracted the attention of many researchers. Zinc mercury thiocyanate (ZMTC), cadmium mercury thiocyanate (CMTC), zinc cadmium thiocyanate (ZCTC) and manganese mercury thiocyanate (MMTC) are some of the crystals belonging to this category. ZMTC and CMTC are found to have all the good characteristics such as crystallizing in a 
noncentrosymmetric space group $\mathrm{I} \overline{4}$, colourless and high thermal stability [1]. ZMTC and CMTC are excellent nonlinear materials capable of second harmonic generation of $\mathrm{Nd}$ : YAG laser radiation of wavelength $1064 \mathrm{~nm}$ [2, 3]. Intracavity-frequency-doubling of a $946 \mathrm{~nm} \mathrm{Nd:}$ YAG laser with CMTC crystal was reported by Changqiang Wang et al. [4] and generation of blue-violet light at room temperature with GaAlAs diode laser was reported by Yuan et al. [5]. These crystals can not be grown from melt as they undergo decomposition before melting and can be obtained only from solution. Some of the doped crystals of the bimetallic thiocyanate complexes are also found to exhibit excellent nonlinear optical properties [6]. Pure and magnesium doped CMTC single crystals of considerably large size were obtained from silica gel in our laboratory.

A comparative study of pure and Mg doped CMTC crystal is reported in this paper. Growth of magnesium doped CMTC crystal is discussed in detail.

\section{GEL GROWTH}

Single diffusion method was used for the growth of pure and $\mathrm{Mg}$ doped CMTC single crystals in silica gel. High grade analytical reagents of mercury(II) chloride, ammonium thiocyanate, cadmium chloride and magnesium chloride were used. Mercury(II) chloride and ammonium thiocyanate together were taken as the inner reagent and cadmium chloride was used as the outer reagent. The reaction that takes place for the growth of pure CMTC is

$$
\mathrm{CdCl}_{2}+4 \mathrm{NH}_{4} \mathrm{SCN}+\mathrm{HgCl}_{2} \longrightarrow \mathrm{CdHg}(\mathrm{SCN})_{4}+4 \mathrm{NH}_{4} \mathrm{Cl}
$$

The following procedure was followed for the growth of Mg doped CMTC crystals. As reported by Henisch, stock solution of sodium meta silicate was prepared by adding 244 grams of sodium meta silicate $\left(\mathrm{Na}_{2} \mathrm{SiO}_{3} 9 \mathrm{H}_{2} \mathrm{O}\right)$ to $500 \mathrm{ml}$ of distilled water [7]. Stock solution $(7.5 \mathrm{ml})$ was diluted with equal quantity of distilled water $(7.5 \mathrm{ml})$ and its $\mathrm{pH}$ was adjusted to 3.4 using ExcelaR grade glacial acetic acid of purity 99.8. This stock solution of $\mathrm{pH} 3.4$ was mixed with a mixture of $32 \mathrm{ml}$ aqueous solution of $0.2 \mathrm{M} \mathrm{HgCl}_{2}$ and $16 \mathrm{ml}$ aqueous solution of $4 \mathrm{M} \mathrm{NH} \mathrm{NCN}_{4}$ and allowed to gel in test tubes of length $15 \mathrm{~cm}$ and diameter $1.5 \mathrm{~cm}$. It took nearly 48 hours for gelation to be complete. Then the outer reagent (a mixture of $90 \%$ aqueous solution of $3 \mathrm{M} \mathrm{CdCl}_{2}$ and $10 \%$ aqueous solution of $3 \mathrm{M} \mathrm{MgCl}_{2}$ ) was added on to the top of the gel using a pipette. Due to the diffusion of the outer reagent in to the gel medium and its reaction with the inner reagents, crystals started growing.

Colorless transparent crystals of considerable size were recovered within 30 days at an ambient temperature of about $30^{\circ} \mathrm{C}$. Figures 1 and 2 show the photographs of pure and $\mathrm{Mg}$ doped CMTC single crystals inside and outside the gel medium respectively. The presence of dopant in the 
crystal has been quantitatively computed from the inductively coupled plasma analysis as 0.0093 $\%$ using the formula:

Weight $\%=\left(\right.$ Concentration in ppm or $\mathrm{mg} / \mathrm{L} \mathrm{X}$ Volume in $\left.\mathrm{ml} \mathrm{X} \mathrm{10^{-4 }}\right) /$ Weight of the sample in $\mathrm{g}$ Concentration of $\mathrm{Mg}=0.184 \mathrm{mg} / \mathrm{L}$, Weight of the sample $=0.09866 \mathrm{~g}$, Volume $=50 \mathrm{ml}$.
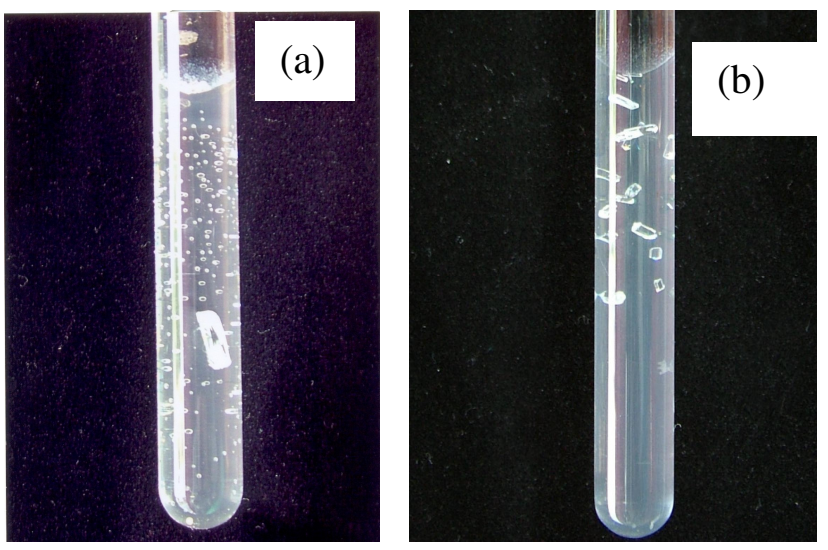

Fig. 1. Single crystals in gel medium (a) Pure CMTC (b) Mg doped CMTC.
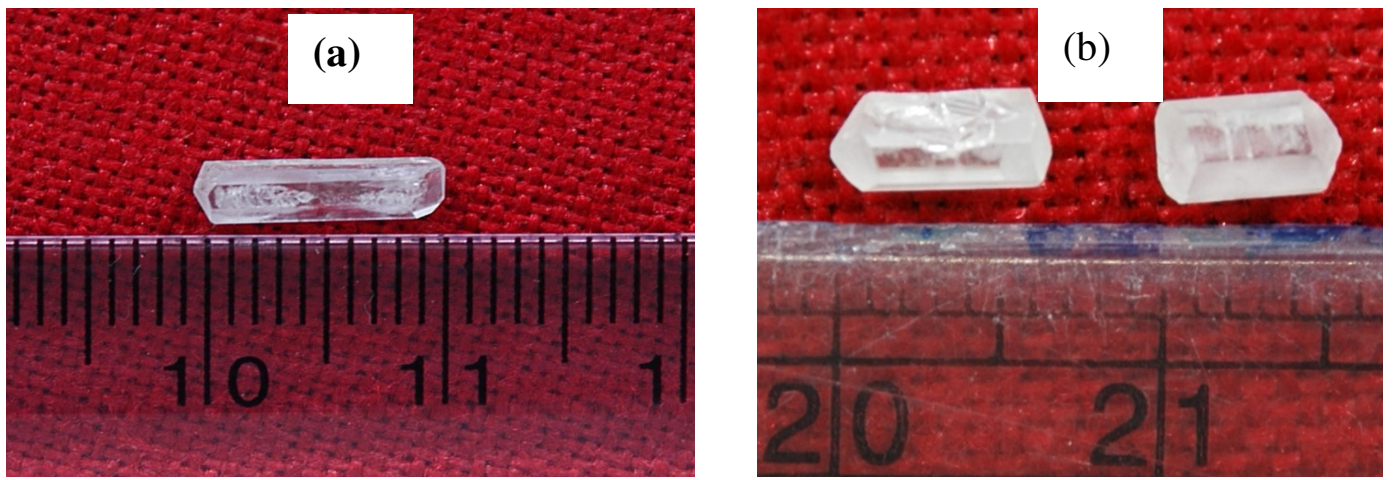

Fig. 2. As grown single crystals (a) Pure CMTC (b) Mg doped CMTC.

\section{CHARACTERIZATIONS}

The experimental details of the characterizations carried out for the gel-grown single crystals are briefly described below.

The powder X-ray diffractometry analysis was performed with a graphite monochromated $\mathrm{CuK} \alpha$ radiation. Single crystal X-ray diffraction analysis was performed on ENRAF NONIUS FR590 diffractometer with Mo K $\alpha$ radiation of wavelength $0.7170 \AA$. 
The crystalline perfection of the grown crystals was analyzed with a multicrystal X-ray diffractometer developed at National Physical Laboratory (NPL) [8]. In this system, a fine focus $(0.4$ x $8 \mathrm{~mm} ; 2 \mathrm{~kW}$ Mo) X-ray source energized by a well-stabilized Philips X-ray generator (PW 1743) was employed. The well-collimated and monochromated $\mathrm{MoK} \alpha_{1}$ beam obtained from the three monochromator $\mathrm{Si}$ crystals set in dispersive $(+,-,-)$ configuration has been used as the exploring X-ray beam. This arrangement improves the spectral purity $\left(\Delta \lambda / \lambda<<10^{-5}\right)$ of the $\mathrm{MoK} \alpha_{1}$ beam. The divergence of the exploring beam in the horizontal plane (plane of diffraction) was estimated to be $<<3$ arc sec. The specimen crystal is aligned in the $(+,-,-,+)$ configuration. Due to dispersive configuration, though the lattice constant of the monochromator crystal and the specimen are different, the unwanted dispersion broadening in the diffraction curve of the specimen crystal is insignificant. The specimen can be rotated about a vertical axis, which is perpendicular to the plane of diffraction, with minimum angular interval of 0.5 arc sec. The diffracted intensity is measured using a scintillation counter and is mounted with its axis along a radial arm of the turn table. The rocking or diffraction curves were recorded by changing the glancing angle (angle between the incident X-ray beam and the surface of the specimen) around the Bragg diffraction peak position $\theta_{\mathrm{B}}$ starting from a suitable arbitrary glancing angle (denoted as zero).

The Fourier transform infra red spectra were recorded for the powdered samples with AVATAR 330 FTIR spectrometer in the region $4000--400 \mathrm{~cm}^{-1}$ using the $\mathrm{KBr}$ pellet technique.

The transmission spectra were recorded for the samples in solution state using HITACHI U-2800 double beam spectrophotometer in the wavelength range 200-1000 $\mathrm{nm}$.

To investigate the thermal stability of the crystals, thermogravimetric and differential thermal analyses (TG/DTA) were carried out in nitrogen atmosphere in the temperature range 50-$1100^{\circ} \mathrm{C}$ using an STA 409PC instrument.

Vickers microhardness studies were carried out using an MH-112 Vickers microhardness tester (Japan). Kurtz powder test was carried out to determine its second harmonic generation efficiency in comparison with urea.

\section{RESULTS AND DISCUSSION}

\subsection{X-ray Diffraction Analysis}

The powder X-ray diffraction patterns of pure and Mg doped CMTC crystals are shown in Fig. 3. The diffraction planes are identified and indexed. The single crystal X-ray data collected using ENRAF NONIUS FR590 diffractometer were solved by the direct method and refined by the full matrix least square technique using SHELHL program. The crystal structure of Mg doped 
CMTC is determined to be tetragonal revealing that the incorporation of the dopant has not changed the structure of the parent crystal. The variations in intensity of peaks, lattice parameters and increase in cell volume attribute to the incorporation of the dopant in the crystal. The lattice parameters of pure and Mg doped CMTC crystals are $\mathrm{a}=\mathrm{b}=11.473 \AA, \mathrm{c}=4.250 \AA, \propto=\beta=\gamma=90^{\circ}$ and $\mathrm{V}=559.43 \AA^{3}$ (Pure CMTC). $\mathrm{a}=\mathrm{b}=11.445 \AA, \mathrm{c}=4.206 \AA, \propto=\beta=\gamma=90^{\circ}$ and $\mathrm{V}=550.94 \AA^{3}$ (Mg doped CMTC).
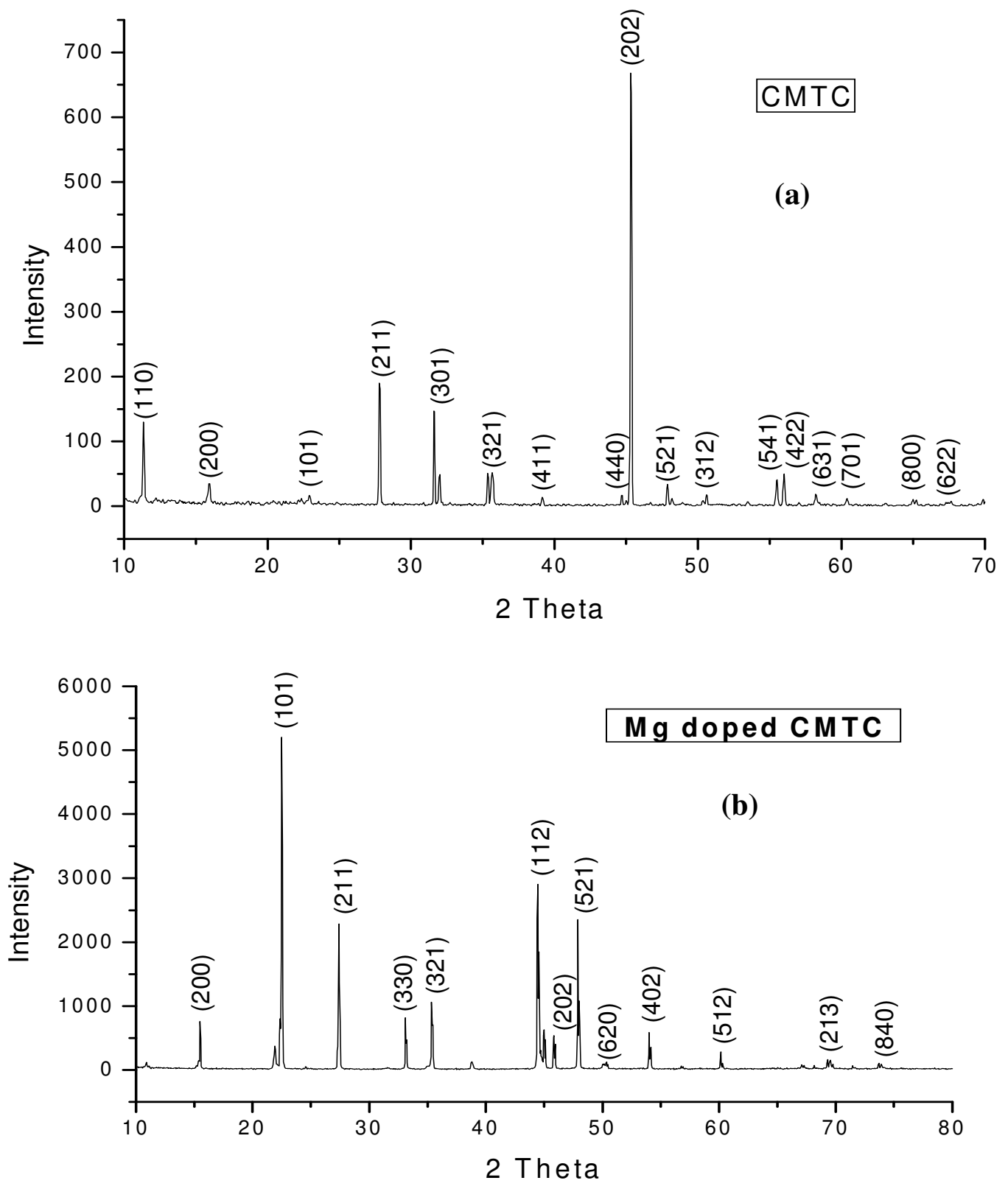

Fig. 3. Powder X-ray diffraction pattern (a) Pure CMTC (b) Mg doped CMTC. 


\subsection{X-ray Rocking Curve Studies}

Fig. 4 shows the high-resolution diffraction curves recorded for $\left(\begin{array}{lll}2 & 0 & 0\end{array}\right)$ plane of pure CMTC and (1 $\left.0 \begin{array}{ll}1 & 0\end{array}\right)$ plane of Mg doped CMTC with a multicrystal X-ray diffractometer in symmetrical Bragg geometry. Before recording the diffraction curves the specimen surfaces were prepared by lapping and polishing and then chemically etched by a non-preferential chemical, mixed with water and acetone in 1:2 ratios. The diffraction curve for pure CMTC contains two additional peaks which are 16 and 37 arc sec away from the main peak. These two additional peaks correspond to two internal structural very low angle (tilt angle $\leq 1$ arc min) boundaries [9] whose tilt angles (misorientation angle between the two crystalline regions on both sides of the structural grain boundary) are 16 and 21 arc sec from their adjoining regions. The FWHM (full width at half maximum) of the main peak and the low angle boundaries are respectively 20, 17 and 21 arc sec. Though the specimen contains very low angle boundaries, the relatively low angular spread of around 150 arc sec of the diffraction curve and the low FWHM values show that the crystalline perfection is quite good. In the case of $\mathrm{Mg}$ doped CMTC, the solid line in the figure is well fitted with the experimental points represented by the open circles. The specimen does not contain any internal structural grain boundaries. The FWHM (full width at half maximum) of the sharp main peak is very low (32 arc sec) showing that the crystalline perfection is very good revealing that it is a suitable candidate for nonlinear applications.
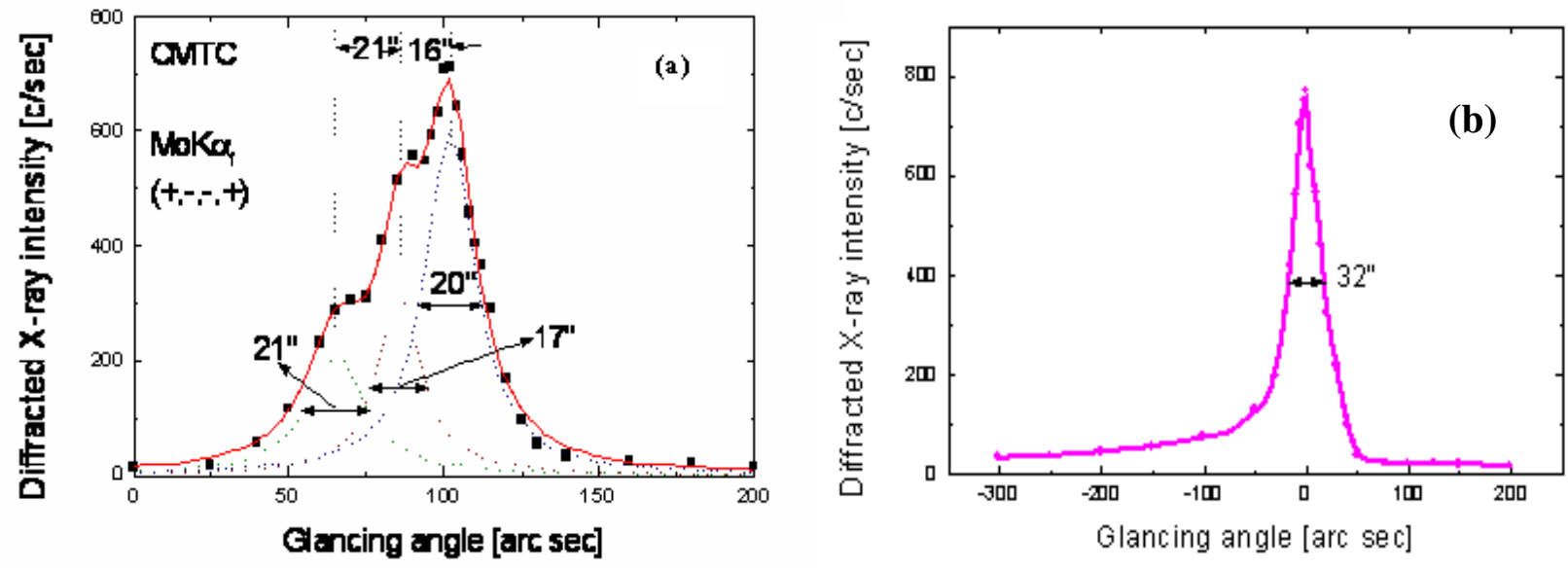

Fig. 4. X-ray rocking curve recorded for (202) plane (a) Pure CMTC (b) Mg doped CMTC.

\subsection{Fourier Transform Infrared Spectroscopic Studies}

The Fourier transform infrared spectral analysis is a technique in which almost all functional groups in a molecule absorb characteristic frequencies. The absorption of IR radiation causes 
bending and stretching vibrations. The most important range being $4000-400 \mathrm{~cm}^{-1}$, FTIR spectrum was recorded in that range using $\mathrm{KBr}$ pellet technique. Fig. 5 shows the FTIR spectra of pure and $\mathrm{Mg}$ doped CMTC. The absorption peaks and the assignment of frequencies are shown in Table 1.
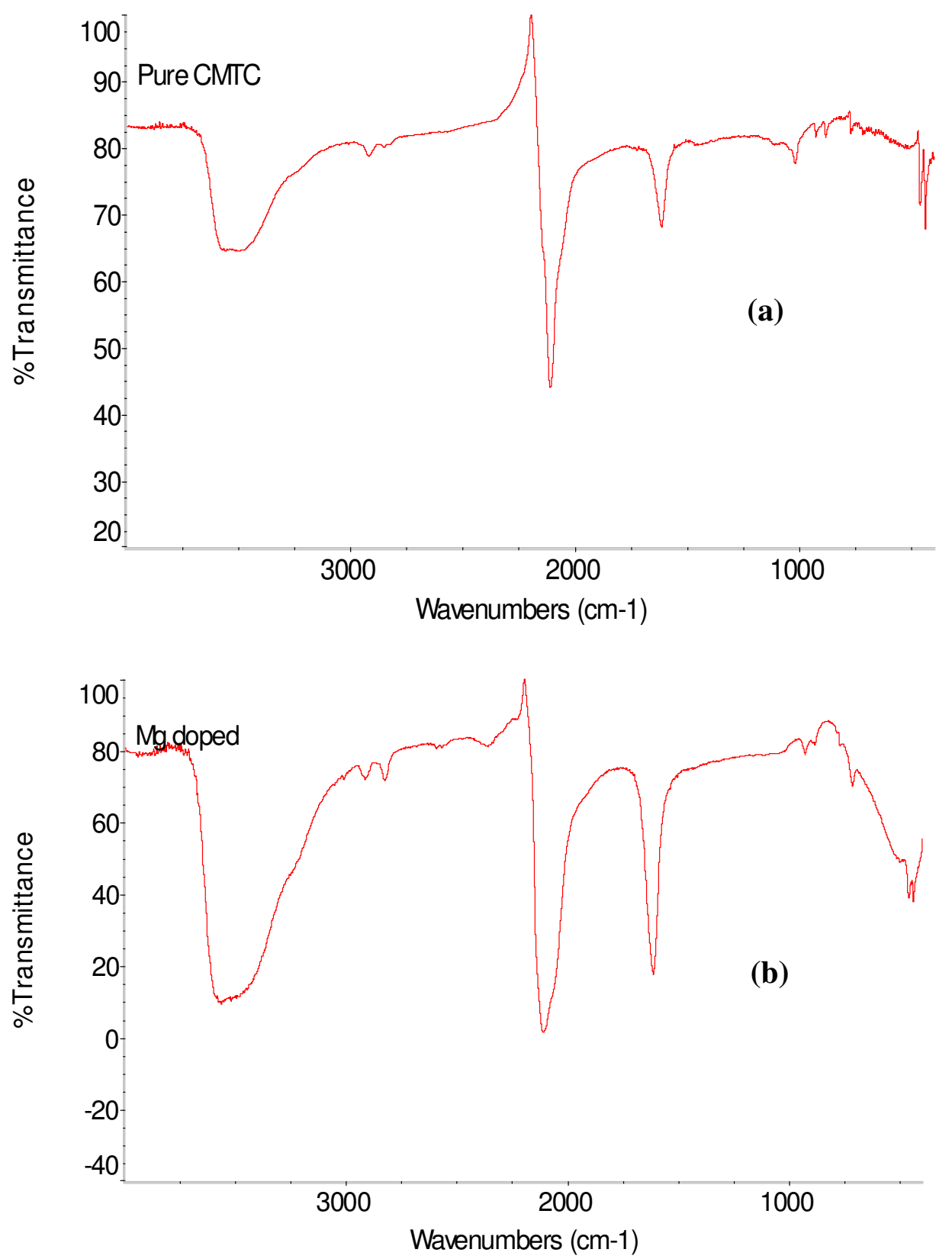

Fig. 5. FTIR spectrum (a) Pure CMTC (b) Mg doped CMTC. 
Table 1. FTIR bands of pure and Mg doped CMTC.

$\begin{array}{ccccc}\text { Crystals } & \boldsymbol{v}_{C N} & \boldsymbol{v}_{C S} & \boldsymbol{\delta}_{\text {NCS }} & \mathbf{2} \boldsymbol{\delta}_{\text {NCS }} \\ & & & & \\ \text { CMTC } & 2110.24 & 767.75 & 463.18,440.86 & 927.73,883.33 \\ \text { Mg doped } & 2111.42 & 715.42 & 459.69,439.83 & 928.84,874.01\end{array}$

It is well known that $\mathrm{CN}$ stretching vibration often lies higher than $2100 \mathrm{~cm}^{-1}$, CS stretching lies between $860-780 \mathrm{~cm}^{-1}$ (N-bonding) or 720-690 $\mathrm{cm}^{-1}$ (S-bonding) and SCN bending vibration lies near $480 \mathrm{~cm}^{-1}$ (N-bonding) or $420 \mathrm{~cm}^{-1}$ (S-bonding) [10, 11]. From the table it is obvious that $v_{\mathrm{CN}}$ is higher than $2100 \mathrm{~cm}^{-1}$ in both the cases and the CS vibration is observed around $770 \mathrm{~cm}^{-1}$ in pure CMTC and around $715 \mathrm{~cm}^{-1}$ in Mg doped CMTC indicating S-bonding in the complex in $\mathrm{Mg}$ doped CMTC crystal. The intense broad band observed around $3500 \mathrm{~cm}^{-1}$ and the weak band around $1650 \mathrm{~cm}^{-1}$ in the IR spectra of the pure and $\mathrm{Mg}$ doped crystals correspond to stretching and bending modes of water respectively.

\subsection{Transmission Spectral Analysis}

The transmission spectra were recorded in solution state using the ethanol-water (ratio 1:1) solvent. The powdered samples were dissolved in the mixed solvent $(803 \mathrm{mmol} / \mathrm{l})$ and transmission spectra were recorded using HITACHI model U-2800 double beam spectrophotometer in the wavelength range $200-1000 \mathrm{~nm}$. The spectra of pure and doped CMTC are shown in Fig. 6. The transparency cut offs are at 311 and $315 \mathrm{~nm}$ respectively in pure and Mg doped CMTC.

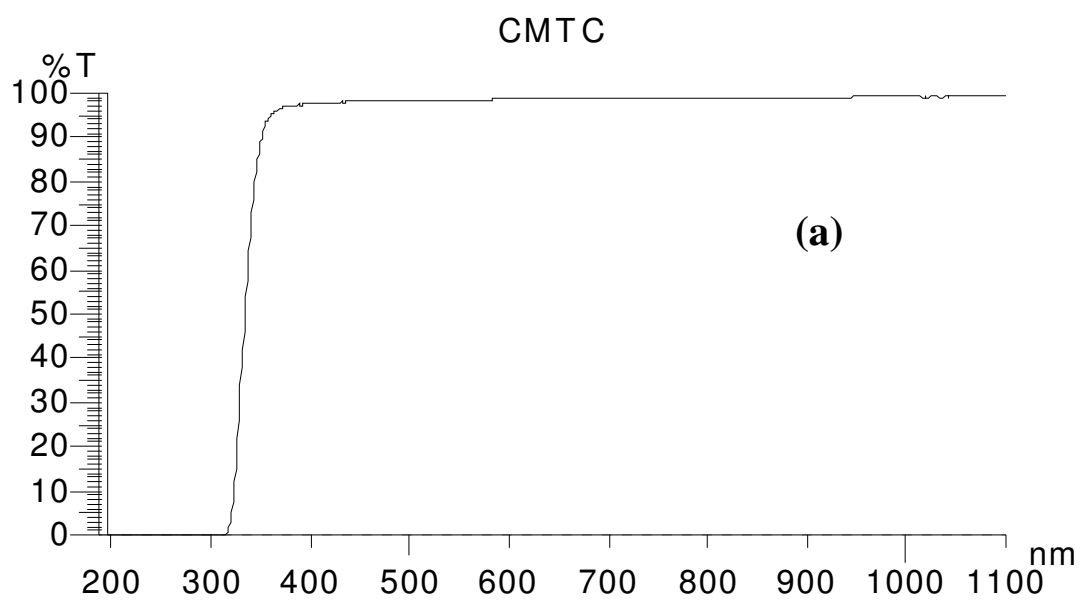




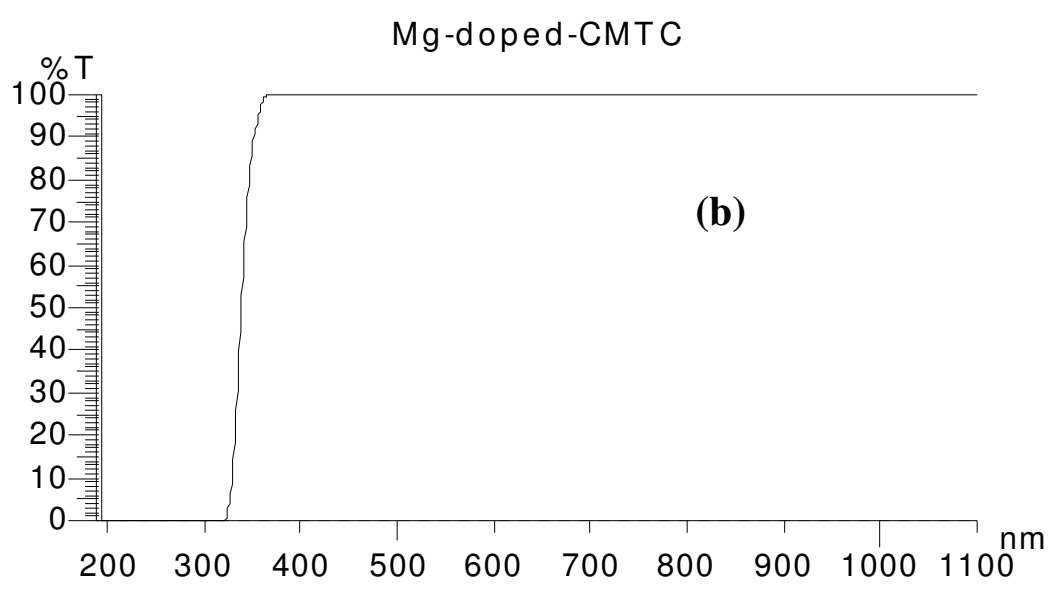

Fig. 6. Transmission spectrum (a) Pure CMTC (b) Mg doped CMTC.

\subsection{Thermal Analysis}

Thermogravimetric and differential thermal (TG/DTA) analyses were carried out for the grown crystals in nitrogen atmosphere at a heating rate of $20^{\circ} \mathrm{C} / \mathrm{min}$. Fig. 7 shows the TG/DTA curves of pure and Mg doped CMTC. The losses of masses at different stages in pure and doped CMTC are shown in Table 2.

Table 2: Thermal analysis (TG) data of pure and Mg doped CMTC.

Pure CMTC

Tempe. Range Mass loss

$272-298^{\circ} \mathrm{C}$
$298-668^{\circ} \mathrm{C}$
$668-1099^{\circ} \mathrm{C}$
Residue
$8.48 \%$

$65.71 \%$

$4.49 \%$

$19.22 \%$
Mg doped CMTC

Temp. Range Mass loss

$274-324^{\circ} \mathrm{C} \quad 8.84 \%$

$324-600^{\circ} \mathrm{C} \quad 56.87 \%$

$600-792^{\circ} \mathrm{C} \quad 7.47 \%$

$911-1099^{\circ} \mathrm{C} \quad 1.4 \%$

Residue $\quad 23.96 \%$ 

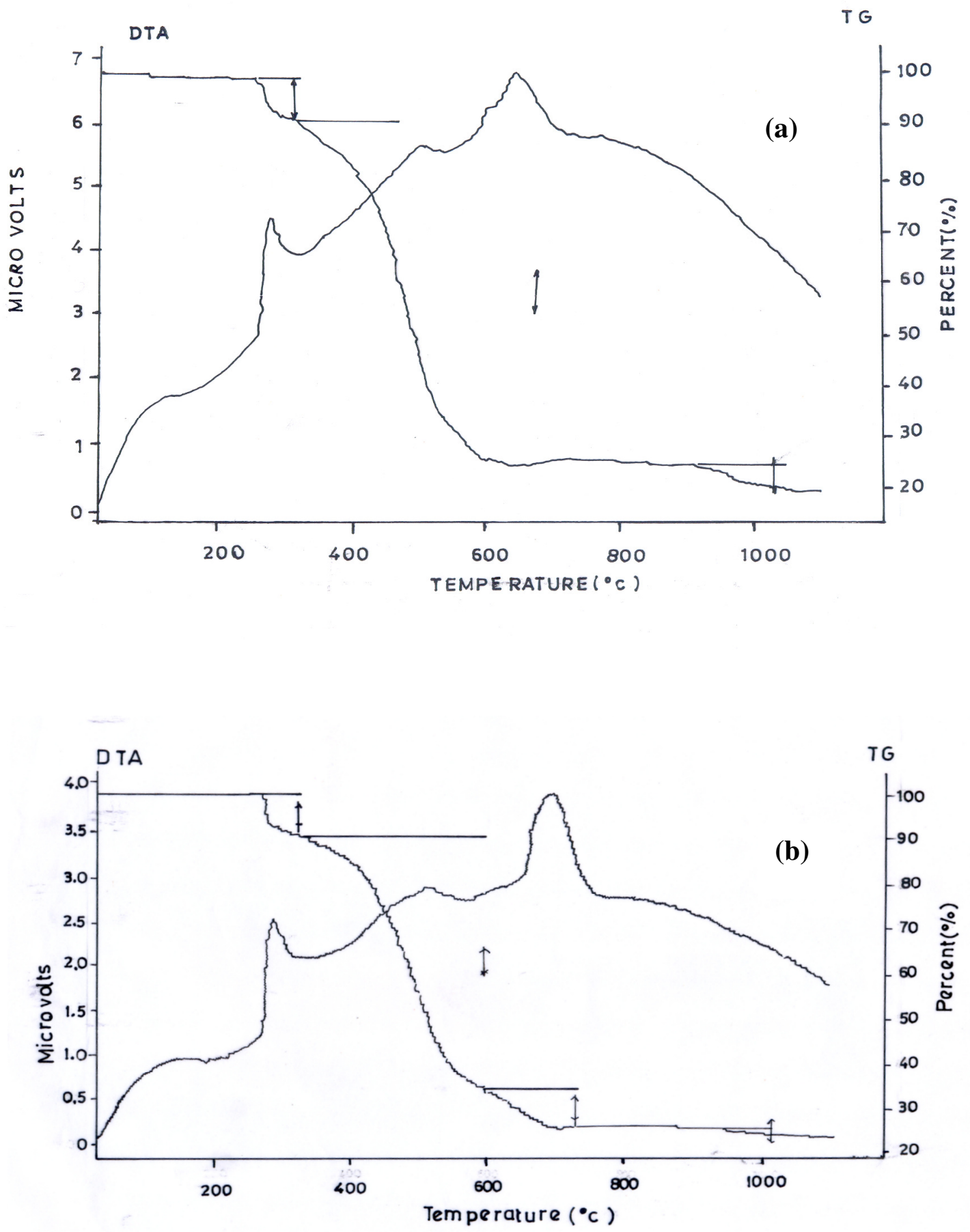

Fig. 7. TG/DTA curves (a) Pure CMTC (b) Mg doped CMTC. 
The observed mass loss $(2.1 \%$ for pure and $1.46 \%$ for $\mathrm{Mg}$ doped) below the decomposition point is due to dehydration. CMTC crystals are thermally stable up to $272^{\circ} \mathrm{C}$ and decomposition starts only at $272^{\circ} \mathrm{C}$. The TG curve of pure CMTC shows three steps. At $272^{\circ} \mathrm{C}$, breakdown of the three-dimensional steric structure takes place with the evaluation of $7 / 8(\mathrm{CN})_{2}$ (cyanogen) corresponding to the mass loss in the first step. The major mass loss in the second step corresponds to the evaluation of $1 / 8(\mathrm{CN})_{2}, 1 / 4 \mathrm{~N}_{2}$ (nitrogen) gas, $2 \mathrm{CS}_{2}$ (carbon disulphide) and sublimation of $\mathrm{Hg}$. In the third step, the temperature being greater than $767^{\circ} \mathrm{C}$ (the boiling point of cadmium) sublimation of cadmium initiates and at $1099^{\circ} \mathrm{C}, 93.24 \%$ of cadmium remains as residue. The DTA curve shows two significant exothermic peaks at $280^{\circ} \mathrm{C}$ and $646^{\circ} \mathrm{C}$ corresponding to the evaluation of volatile gases corresponding to steps one and two respectively in the TG curve

The thermogram shows that $\mathrm{Mg}$ doped CMTC crystals are thermally stable up to $274^{\circ} \mathrm{C}$ and decomposition starts only at $274^{\circ} \mathrm{C}$. The TG curve shows four steps. In the first step, breakdown of three-dimensional steric structure occurs with the evaluation of volatile gases. The major mass loss $(56.87 \%)$ in the second step corresponds to the sublimation of $\mathrm{Hg}$. In the third step along with the evaluation of volatile gases sublimation of cadmium initiates which continues in the fourth step. At $1099^{\circ} \mathrm{C}$ cadmium metal along with magnesium remains as residue $(23.96 \%)$. This is confirmed by an increase in the residue percentage. The observed high decomposition temperature $\left(274^{\circ} \mathrm{C}\right)$ shows the greater thermal stability of the Mg doped CMTC crystals. The DTA curve shows two significant exothermic peaks at $284^{\circ} \mathrm{C}$ and $690^{\circ} \mathrm{C}$ corresponding to steps one and three of the TG curve.

\subsection{Hardness Test}

Vickers hardness indentations were made on the flat polished face of the crystals at room temperature for loads 10, 25 and $50 \mathrm{~g}$ using Vickers hardness tester fitted with Vickers diamond indenter and attached to an incident light microscope. Since crack initiation and material chipping became significant beyond $50 \mathrm{~g}$ of the applied load, hardness test could not be carried out above this load. The lengths of the two diagonals of the indentations were measured and the Vickers hardness number was computed using the formula

$$
\mathrm{H}_{\mathrm{V}}=1.8544 \mathrm{P} / \mathrm{d}^{2}
$$

Where $\mathrm{H}_{\mathrm{V}}$ is the Vickers hardness number in $\mathrm{kg} / \mathrm{mm}^{2}, \mathrm{P}$ is the indenter load in $\mathrm{kg}$ and $\mathrm{d}$ is the diagonal length of the impression in $\mathrm{mm}$. The variation of $\mathrm{H}_{\mathrm{V}}$ with load and Plot of $\log \mathrm{P}$ vs. $\log$ $\mathrm{d}$ for pure and $\mathrm{Mg}$ doped CMTC are shown in Fig. 8. In the case of pure CMTC $\mathrm{H}_{\mathrm{V}}$ increases with increase in $\mathrm{P}$ and Meyer's index number ' $\mathrm{n}$ ' is estimated as 2.89 [12]. The Vickers 
hardness number of Mg doped CMTC crystal decreases with increase of load and the Meyer's index number ' $\mathrm{n}$ ' is estimated from the slope as 1.52. Hence we can say that Pure CMTC belongs to soft material category and Mg doped CMTC belongs to the hard material category $[13,14]$.
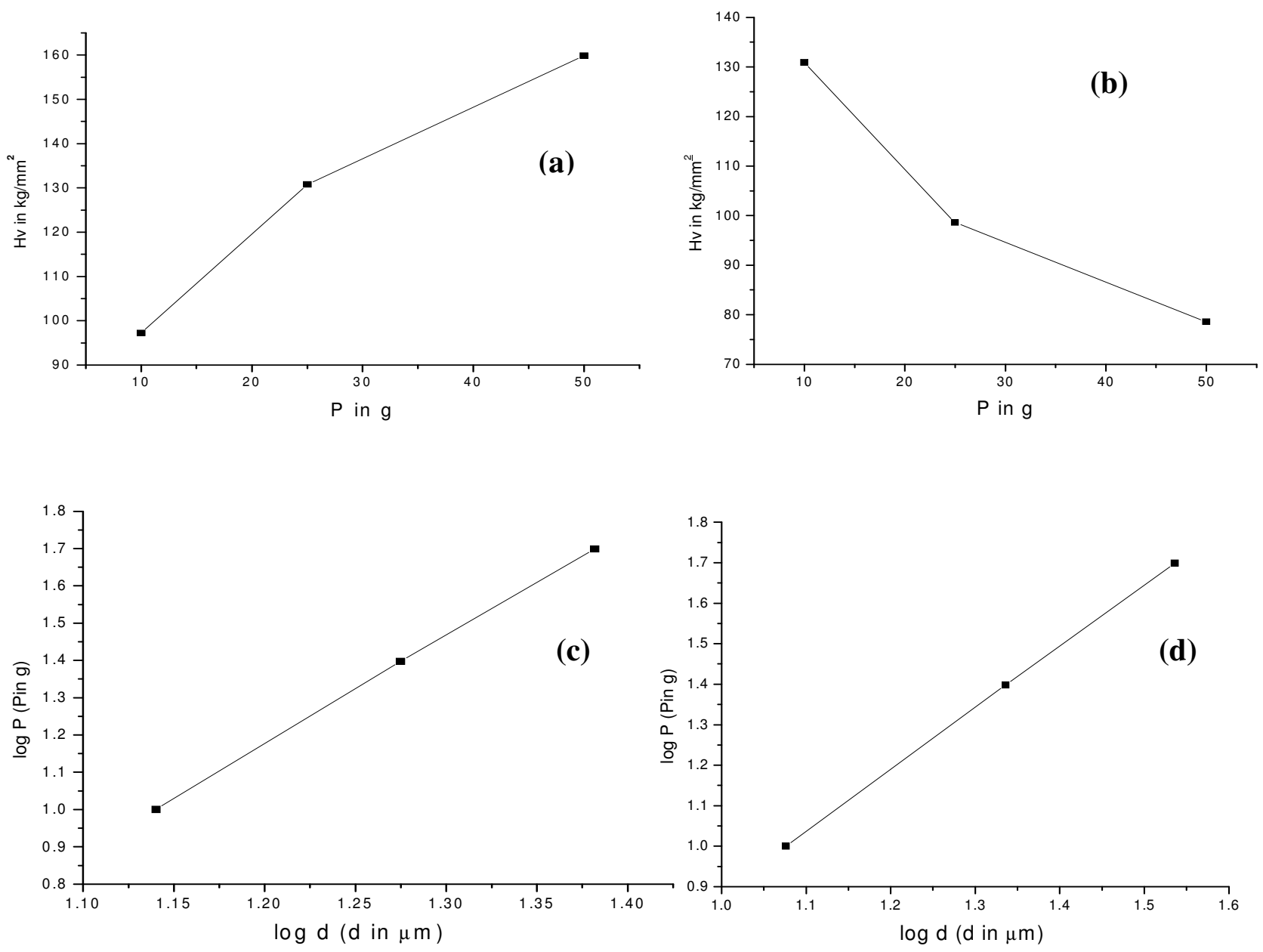

Fig. 8 (a). Plot of $\mathrm{H}_{\mathrm{V}}$ vs. $\mathrm{P}$ for pure CMTC.

(b). Plot of $\mathrm{H}_{\mathrm{V}}$ vs. P for $\mathrm{Mg}$ doped CMTC.

(c). Plot of $\log \mathrm{P}$ vs. $\log \mathrm{d}$ for pure CMTC.

(d). Plot of $\log$ P vs. $\log \mathrm{d}$ for Mg doped CMTC.

\subsection{Powder Kurtz Method}

A preliminary study on the second harmonic generation efficiency of the crystal with reference to urea was carried out by powder technique developed by Kurtz and Perry [15]. In this experiment Q-switched Nd-YAG laser with the first harmonic output of $1064 \mathrm{~nm}$ was used. The 
crystal was ground to homogeneous powder and tightly packed in a micro capillary tube and mounted in the path of the laser beam of pulse energy $5 \mathrm{~mJ}$. The SHG was confirmed by the emission of green light $(\lambda=532 \mathrm{~nm})$ collected by photomultiplier tube and displayed on the oscilloscope. SHG signals of intensity $930 \mathrm{mV}$ and $864 \mathrm{mV}$ were recorded for pure and $\mathrm{Mg}$ doped CMTC while that for urea was $150 \mathrm{mV}$. The second harmonic generation efficiencies of pure and Mg doped CMTC are respectively found to be 6.2 and 5.8 times greater than that of urea.

\section{CONCLUSION}

Mg doped CMTC single crystals of good optical quality have been obtained from silica gel in an acidic medium with gel technique by the process of diffusion. The reagents required in this case are magnesium chloride, cadmium chloride, mercury(II) chloride and ammonium thiocyanate. The X-ray diffraction study reveals that Mg doped CMTC single crystals formed in silica gel are very much crystalline in nature having tetragonal structure. HRXRD studies reveal that the crystalline perfection of the doped crystal is extremely good. High thermal stability of the crystal is confirmed by TG/DTA analysis and Vickers microhardness studies show that the mechanical property of the crystal is good. In comparison with urea its second harmonic generation efficiency is greater thus making it an excellent candidate for nonlinear applications.

\section{ACKNOWLEDGEMENT}

The authors thank the Management of VIT University, Vellore for their constant encouragement and support. The authors are grateful to Dr.G.Bhagavannarayana, Materials Characterization Division, National Physical Laboratory, The Head, Materials Characterization Division, NPL and The Director, NPL for the support to carry out the HRXRD studies.

\section{REFERENCES}

[1] X.Q.Wang et al. Mater.Res.Bull. 37, P1859-1871 (2002).

[2] J.G.Bergman, J.J.Mcfee, G.R.Crane, Mater. Res. Bull. 5, P913 (1970).

[3] W.Sturmer, U.Deserno, Phys.Lett. A 32, P539 (1970).

[4] Changqiang Q. Wang et al. Optics \&Laser Technol. 30, P291-293 (1998).

[5] D.R.Yuan et al. Appl. Phys. Lett. 70, P544 (1997).

[6] G.P.Joseph et al. Cryst.Res.Technol. 42, P295-299 (2007).

[7] Heinz K.Henisch, Crystals in Gels and Liesegang Rings, Cambridge University press, Cambridge, 1988.

[8] Krishan Lal and G. Bhagavannarayana, J. Appl. Cryst. 22, P209-215 (1989).

[9]G. Bhagavannarayana et al. Appl. Cryst. 38, P768-771 (2005).

[10]K.Nakamoto, Infrared and Raman spectra of Inorganic and co-ordination compounds, Third 
edition, Wiley, New York, 1978.

[11]R.M.Silverstein and F.X.Webster, Spectroscopic identification of organic compounds, sixth edition, Wiley, New York, 1998.

[12] P.Nisha Santha Kumari, S.Kalainthan, N.Arunai Nambi Raj, Materials Letters, 62, P305308 (2008).

[13]E.M.Onitsch, Mikroscopia, 2, P131 (1947).

[14]M.Hanneman, Metall. Manchu. 23, P135 (1941).

[15]S.K.Kurtz and T.T.Perry, J. Appl. Phys. 39, P3798 (1968). 